\title{
Humour(s) et humeur(s), dossier coordonné par Rosalia Bivona
}

Ilaria Vitali

\section{(2) OpenEdition}

1 Journals

\section{Edizione digitale}

URL: http://journals.openedition.org/studifrancesi/6122

DOI: $10.4000 /$ studifrancesi.6122

ISSN: 2421-5856

\section{Editore}

Rosenberg \& Sellier

\section{Edizione cartacea}

Data di pubblicazione: 1 mai 2011

Paginazione: 221-222

ISSN: 0039-2944

\section{Notizia bibliografica digitale}

Ilaria Vitali, «Humour(s) et humeur(s), dossier coordonné par Rosalia Bivona», Studi Francesi [Online],

163 (LV | I) | 2011, online dal 30 novembre 2015, consultato il 09 janvier 2021. URL: http://

journals.openedition.org/studifrancesi/6122 ; DOI: https://doi.org/10.4000/studifrancesi.6122

Questo documento è stato generato automaticamente il 9 janvier 2021.

\section{(c) (i) (9)}

Studi Francesi è distribuita con Licenza Creative Commons Attribuzione - Non commerciale - Non opere derivate 4.0 Internazionale. 


\title{
Humour(s) et humeur(s), dossier coordonné par Rosalia Bivona
}

\author{
Ilaria Vitali
}

\section{NOTIZIA}

Humour(s) et humeur(s), dossier coordonné par Rosalia BIVONA, «Expressions

maghrébines», vol. 7, n. 2, inverno 2008, pp. 215.

1 Humour(s) et humeur(s), sin dal titolo, questo volume di «Expressions Maghrébines» fa convergere l'attenzione sulla natura molteplice del comico. La curatrice, Rosalia Bivona, ne mette in luce la produttiva problematicità fin dall'introduzione, che annuncia la ricchezza di un tema declinato in maniera diversa in ognuno degli articoli che compongono il volume. Che cos'è l'humour? E cosa caratterizza, in particolare, l'humour maghrebino? Sono queste le domande alla base di questo numero tematico, lanciate nell'introduzione e subito riprese dall'articolo di apertura, dal titolo De quelques manifestations de l'humour dans la littérature maghrébine d'expression française; in questo primo studio, Abdellah HAммоUті traccia una rapida panoramica delle diverse manifestazioni dell'umorismo nella letteratura maghrebina, per poi metterne in rilievo la specificità: l'humour maghrebino non si limiterebbe, secondo l'autore, a denunciare un determinato contesto sociale e culturale, ma tratterebbe soprattutto temi di ordine politico, filosofico, etico ed estetico. Spetta a Bernard URBANI il compito di entrare nello specifico, attraverso l'analisi di un celebre romanzo di Ben Jelloun che si colloca tra il tragico e il comico. Nell'articolo, dal titolo Entre humour et dérision: "Moha le fou Moha le sage" de Tahar Ben Jelloun, lo studioso sonda la messa in discussione dell'ordine religioso e politico dell'universo maghrebino, in particolare del Marocco, a cui da anni Ben Jelloun ha abituato il suo lettore. Sotto le spoglie di «jongleur de mots», quasi di saltimbanco, nomade e «folle», Moha rivela infatti attraverso l'umorismo una profonda volontà di denuncia e contestazione del potere. Nel terzo articolo, Modalités d'énonciation de l'humour et de la dérision dans "Un été de cendres" d'Abdelkader Djemaï, 
Faouzia BENDJELID affronta invece l'analisi di un romanzo che, a prima vista, niente sembra aver a che vedere con l'umorismo. Pubblicato nel 1995, Un été de cendres s'iscrive infatti nel «decennio nero» dell'Algeria, fatto di violenze e attentati. Tuttavia, come mostra la studiosa, nel contesto integralista in cui si colloca inevitabilmente la narrazione di Djemaii, la «derisione» diventa una potente arma-usata in maniera sistematica - di analisi e demistificazione. Lo studio del continuo intreccio di comico e tragico passa qui attraeverso l'analisi (non scontata) della relazione "triangolare» autore/testo/lettore. A seguire, Mourad YeLLES propone uno studio dell'umorismo nell'universo letterario di Mohamed Dib. In questo articolo, dal titolo De la danse des mots au sourire du chat: l'humour dibien entre "nûkta" et "nonsense", entra in gioco il concetto di «assurdo»: Dib è infatti «grand amateur de jeux de mots insolites, de fables cocasses ou de paraboles absurdes» (p. 74), situandosi così tra Oriente ed Occidente, e più precisamente tra la tradizione anglosassone del nonsense e quella della nûkta, del folklore comico orientale. Questa originale lettura dell'opera di Dib è seguita da un saggio su un altro grande del panorama letterario maghrebino, Driss Chraïbi, alla cui opera Wafae KARZAZI dedica un'approfondita analisi. Come segnala già il titolo dell'articolo - Les mécanismes de l'ironie dans "Une enquête au pays" de Driss Chraibioggetto dell'indagine è il romanzo Une enquête au pays, primo della saga che vede protagonista l'ispettore Alì (nonché primo polar maghrebino di lingua francese). Nel suo studio, Karzazi sposta l'attenzione dall'umorismo all'ironia, intesa come «forme signifiante de la subversion». (p. 99) Anche l'articolo successivo, La traduction de l'allusion ironique dans "La Fin tragique de Philomène Tralala" de Fouad Laroui, si concentra sulla figura dell'ironia, inadagata tuttavia attraverso lo specchio "deformante» della traduzione: Katrien LIEvors vi analizza infatti le possibilità e i problemi che pone la traduzione dell'ironia, nella fattispecie l'ironia «maghrebina». Oggetto dello studio è la traduzione nederlandese del romanzo di Laroui, con un'analisi dettagliata delle diverse soluzioni traduttologiche messe in atto per rendere le allusioni ironiche del testo di partenza.

2 Fino a questo punto, il numero si interessa unicamente al romanzo, genere privilegiato dalla stragrande maggioranza di autori maghrebini di lingua francese. Tuttavia, anche i racconti per l'infanzia o gli sketch teatrali si prestano, tra gli altri generi, ad un'efficace analisi di humours et humeurs e spetta a Christiane CHAULET-ACHOUR il compito di definirne i contorni. Nell'articolo intitolato En ligne de mire: le foulard et la scène. Humeur et humour «maghrébins», l'autrice, che molti altri studi ha dedicato al «riso» maghrebino, traccia una distinzione ricca di senso tra «rire d'accueil» e «rire d'exclusion», sottolineando come il confine tra accoglienza ed esclusione continuino ad essere, ancora oggi, alla base della creazione maghrebina, che spesso prosegue oltre i confini nazionali, in Francia. Nell'articolo successivo, Rosalia BIVONA analizza il comico attraverso un elemento singolare: il cibo. L'autrice ha del resto già esplorato il rapporto tra mets e mots in un saggio di grande interesse (e fino ad ora unico nel suo genere): $L a$ mensa in scena maghrebina (2005). In questo articolo, la domanda «Le couscous est un plat national français?» è alla base di un'analisi sui rapporti franco-maghrebini visti attraverso lo sguardo irriverente del comico Fellag. Chiude la sezione tematica l'articolo di Carine BOURGET, From "Juif-Arabe" to "Juifs-Arabes": Jews and Arabs in France in Boudjellal's Comic Books, che sposta l'attenzione, dopo romanzo, racconti illustrati e teatro, sulla BD. La studiosa vi analizza la rappresentazione - passata e futura-delle comunità araba e ebraica nello spazio francese attraverso l'opera di Boudjellal. Sono 
infine due i saggi che occupano la sezione «Varia»: nel primo, De la poésie kabyle ancienne, Rachid тітоUсHE sonda l'universo della poesia cabila, relegata fino a non molto tempo fa unicamente all'oralità; nel secondo, Hybridité textuelle chez Abdelkébir Khatibi et Assia Djebar, Névine EL NOSSERY analizza l'opera di due dei maggiori esponenti della letteratura francofona maghrebina attraverso la lente dell'«ibridità testuale». Pur mancando all'appello qualche nome importante del «riso maghrebino» (si pensa, per esempio, a Y.B.), si tratta, nel complesso, di un numero di sicuro interesse per approfondire un tema intrigante e non così frequente nella letteratura in questione. 